\title{
Susceptibility weighted imaging (SWI) for evaluating renal dysfunction in type 2 diabetes mellitus: a preliminary study using SWI parameters and SWI-based texture features
}

\author{
Zhenxing Jiang ${ }^{1}$, Yu Wang ${ }^{1}$, Jiule Ding ${ }^{1}$, Shengnan Yu ${ }^{1}$, Jinggang Zhang ${ }^{1}$, Hua Zhou ${ }^{2}$, Jia Di $^{2}$, Wei Xing ${ }^{1}$ \\ ${ }^{1}$ Department of Radiology, The Third Affiliated Hospital of Soochow University, Changzhou, China; ${ }^{2}$ Department of Nephrology, The Third \\ Affiliated Hospital of Soochow University, Changzhou, China \\ Contributions: (I) Conception and design: Z Jiang, Y Wang, J Ding, W Xing; (II) Administrative support: S Yu, H Zhou, J Di; (III) Provision of study \\ materials or patients: H Zhou, J Di; (IV) Collection and assembly of data: Z Jiang, Y Wang, J Zhang, H Zhou; (V) Data analysis and interpretation: Z \\ Jiang, Y Wang, J Ding; (VI) Manuscript writing: All authors; (VII) Final approval of manuscript: All authors. \\ Correspondence to: Wei Xing. Department of Radiology, The Third Affiliated Hospital of Soochow University, 185 Juqian Street, Changzhou, China. \\ Email: suzhxingwei@126.com.
}

\begin{abstract}
Background: Susceptibility weighted imaging (SWI) could reflect tissue blood oxygen levels, and then whether it could be used to evaluate renal injury remains to be further studied. This study aimed to examine the performance of SWI parameters and SWI-based texture features in evaluating renal dysfunction of type 2 diabetes mellitus (T2DM).

Methods: Forty-five patients with T2DM were included. With the estimated glomerular filtration rate (eGFR), the patients were divided into non-moderate-severe renal injured group (non-msRI, eGFR $>60 \mathrm{~mL} / \mathrm{min} / 1.73 \mathrm{~m}^{2}$ ) and moderate-severe renal injured group (msRI, eGFR $\leq 60 \mathrm{~mL} / \mathrm{min} / 1.73 \mathrm{~m}^{2}$ ). The 3 SWI parameters and 16 SWI-based texture features between non-msRI and msRI were compared. The correlation between the parameters and BUN, Scr was analyzed.

Results: The signal intensity ratio of the medulla to psoas muscle (MPswi) was significantly lower than the signal intensity ratio of the cortex to psoas muscle (CPswi) in non-msRI and msRI group ( $\mathrm{t}=8.619,3.483$, respectively, $\mathrm{P}<0.05)$. MPswi was higher, and the signal intensity ratio of the cortex to the medulla $(\mathrm{CMswi})$, Skewness, Correlation were lower in msRI than in non-msRI $(\mathrm{P}<0.05)$. These parameters showed similar diagnostic efficacies for msRI $(\mathrm{P}>0.05)$, and AUCs were 0.703-0.854. CMswi was an independent protective factor for msRI (OR =0.026, $\mathrm{P}=0.003)$. MPswi and CMswi were correlated with BUN ( $\mathrm{r}=0.416,-0.545$, $\mathrm{P}<0.05)$. CMswi and Correlation were correlated with Scr $(r=-0.645,-0.411, \mathrm{P}<0.05)$.
\end{abstract}

Conclusions: SWI was valuable for assessing renal dysfunction, which may be helpful for the evaluation of moderate-severe renal injured patients with T2DM.

Keywords: Type 2 diabetes mellitus (T2DM); renal insufficiency; magnetic resonance imaging; susceptibilityweighted imaging; image processing, computer-assisted

Submitted Oct 12, 2020. Accepted for publication Dec 02, 2020.

doi: $10.21037 / \mathrm{atm}-20-7121$

View this article at: http://dx.doi.org/10.21037/atm-20-7121

\section{Introduction}

Diabetic nephropathy is a major cause of end-stage renal disease, and nearly $50 \%$ of type 2 diabetes mellitus (T2DM) patients eventually progress to chronic kidney disease (CKD) (1). So far, chronic hypoxia has been considered a standard feature of various progressive kidney diseases, including diabetic nephropathy (2). Hyperglycemia and hyper-filtration of the kidney can cause hypoxia, but the underlying mechanisms are not apparent. The high oxygen demand of the kidney is mainly related to the oxygen 


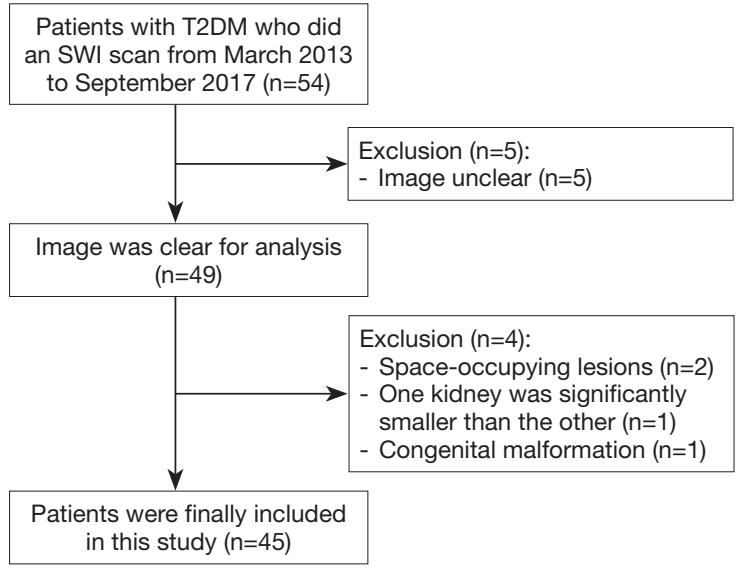

Figure 1 Flowchart of patient selection in this study.

consumption of renal tubular reabsorption (3). It is vital to monitor renal blood oxygen levels noninvasively because it can help understand the kidney oxygenation status and fibrosis process in diabetic nephropathy and to monitor the efficacy of new drugs for diabetic nephropathy. For example, the two drugs recently incorporated into clinical practice, a sodium-glucose cotransporter 2 inhibitors and glucagon-like peptide 1 receptor agonists, can reduce blood glucose, blood pressure, proteinuria, and glomerular filtration rate in T2DM patients, especially in patients with moderate-to-severe diabetic nephropathy (4). Imaging can supply histological blood oxygen level indicators to track drug efficacy precisely.

Susceptibility-weighted imaging (SWI) is an imaging technique that uses the inherent properties of a local magnetic field and enhances image contrast by weighing the phase mask through the amplitude map, which ultimately improves the visibility of various susceptibility sources (5). SWI can reflect tissue blood oxygen levels, and in recent years, it has been increasingly applied in evaluating renal impairment. SWI can monitor the changes of medullary blood oxygen levels after water loading (6) and provide a quantitative evaluation of renal fibrosis in unilateral ureteral obstruction (7). Abnormal lesions with low SWI signals can be observed in patients with delayed graft function after kidney transplantation (8).

Texture analysis quantifies the variability of lesions by analyzing the values and correlations of image pixels. It is beneficial for determining the association between images and pathological processes (9). Texture analysis can quantify tissue heterogeneity and is considered a new biomarker for predicting the decline of renal function in polycystic kidney disease (10). Also, studies have shown that some functional imaging-based texture features can test renal dysfunction (11).

Therefore, the primary aim of this study was to examine the SWI parameters and SWI-based texture features of kidneys in patients with T2DM.

We present the following article in accordance with the STARD reporting checklist (available at http://dx.doi. org/10.21037/atm-20-7121).

\section{Methods}

\section{Study objects}

All procedures performed in this study involving human participants were in accordance with the Declaration of Helsinki (as revised in 2013). The study was approved by the ethics board of the third affiliated hospital of Soochow University (No. 2016-010) and informed consent was taken from all the patients.

From March 2013 to September 2017, a total of 54 consecutive patients diagnosed with T2DM who scheduled for SWI scan from the department of nephrology of the third affiliated hospital of Soochow University were included in the retrospective study. T2DM met the American diabetes association criteria (12).

The inclusion criteria were: (I) patients were diagnosed T2DM with or without CKD; (II) patients were over 18 years old; (III) patients did an SWI scan. Exclusion criteria were: (I) The image was not clear and not suitable for analysis; (II) patients had renal space-occupying lesions (diameter $>1 \mathrm{~cm}$ or the number of single kidney lesions $>5$ ); (III) patients had significant differences in size from the bilateral kidneys (one kidney was significantly smaller than the other); (IV) the patient kidney had congenital malformation.

Ultimately, sample size was determined. Forty-five patients with T2DM who underwent SWI scan were included in this retrospective and single-center study, and the detailed flow chart is shown in Figure 1.Blood urea nitrogen (BUN) and serum creatinine (Scr) were tested within one week before MRI. The estimated glomerular filtration rate (eGFR) was calculated by modification of diet in renal disease $($ MDRD) formula: $175 \times$ creatinine $(\mathrm{mg} / \mathrm{dL})-1.154 \times$ age $($ year $)-0.203 \times 0.742$ (if female) $(13)$. If eGFR was greater than $120 \mathrm{~mL} \cdot \mathrm{min}^{-1} \cdot 1.73 \mathrm{~m}^{-2}$, it was recorded as $120 \mathrm{~mL} \cdot \mathrm{min}^{-1} \cdot 1.73 \mathrm{~m}^{-2}$. With the eGFR level of CKD categories (14), the patients were divided into non- 
Table 1 Sequences and parameters of renal MRI protocols

\begin{tabular}{lcc}
\hline Sequence & T2WI & SWI \\
\hline Imaging plane & Coronal & Transverse \\
Breath technique & Breath-hold & Breath-hold \\
Echo time/repeated time (ms) & $800 / 91$ & $101 / 10$ \\
Field of view $\left(\mathrm{mm}^{2}\right)$ & $380 \times 380$ & $380 \times 285$ \\
Matrix & $256 \times 179$ & $384 \times 250$ \\
Number of slices & 19 & 12 \\
Slice thickness/gap (mm) & $6.5 / 2.0$ & $5.0 / 1.0$ \\
Flip angle $\left({ }^{\circ}\right)$ & 160 & 15 \\
Echo train length & 179 & - \\
Bandwidth (Hz/Px) & 781 & 110 \\
Scan time (s) & 15 & 23 \\
\hline
\end{tabular}

T2WI, T2-weighted imaging; SWI, susceptibility-weighted imaging.

moderate-severe renal injured group (non-msRI, eGFR $>60 \mathrm{~mL} / \mathrm{min} / 1.73 \mathrm{~m}^{2}$ ) and moderate-severe renal injured group (msRI, eGFR $\leq 60 \mathrm{~mL} / \mathrm{min} / 1.73 \mathrm{~m}^{2}$ ).

\section{Magnetic resonance scanning}

All subjects fasted for 4 hours before magnetic resonance imaging (MRI), and none of them took angiotensinconverting enzyme inhibitor or angiotensin receptor blockers before the examination. All scans were performed using a Siemens 3.0 T magnetic resonance scanner (Magnetom Verio; Siemens Healthineers) with a 16-channel phased-array coil. Coronal T2-weighted imaging (T2WI) was conducted first to determine the anatomical localization, followed by cross-sectional SWI scanning. The MRI scanning sequence and parameters are shown in Table 1. Magnitude image, and phase image were retrieved from SWI sequences. For the phase image, the mask was acquired after high-throughput filtering and then weighed with a magnitude image to generate an SWI fusion image (fSWI).

\section{Image analysis}

\section{SWI parameters}

Siemens image-processing software processed the images NUMARIS/4 (version: syngo MR B17) to get susceptibility signal intensity. On the fSWI image, a layer of the right kidney that passes the renal hilum was selected, and three regions of interest (ROIs) were placed on the anterior, medial, and posterior regions of the cortex and medulla. When the boundary between the cortex and medulla was unclear, the cortical ROI was placed close to the edge of the renal cortex, and the medulla ROI was placed in the middle of renal parenchyma (Figure 2). For each patient, the cortical signal intensity (Cswi) and the medullary signal intensity (Mswi) were calculated by averaging the three ROIs. Another ROI was placed on the right psoas major muscle on the same layer to get the psoas muscle signal intensity (Pswi). The ROI placement avoids renal sinus and space-occupying lesions, including cysts. The average size of ROI for all patients was $0.3 \mathrm{~cm} 2$. Cswi and Mswi were divided by Pswi to generate the signal intensity ratio of the cortex to psoas muscle (CPswi) and the signal intensity ratio of the medulla to psoas muscle (MPswi); Cswi divided by Mswi gave rise to the signal intensity ratio of the cortex to the medulla (CMswi).

\section{Texture features}

The texture features were extracted by imaging biomarker explorer, IBEX (15). On the fSWI image, the outline of the entire right kidney was manually marked along the kidney edge on the layer passing right renal hilum. Renal sinus and space-occupying lesions are avoided (Figure 2). The Intensity Histogram and GrayLevelCooccurenceMatrix 25 texture features were extracted at a distance of $\delta=7$ and toward $0^{\circ}$. The following features were included in the analysis: inter quartile range (IQR), Kurtosis, mean absolute deviation (mean $\mathrm{AD}$ ), median absolute deviation (median $\mathrm{AD}), 0.25$ percentile, 0.5 percentile, 0.75 percentile, range, skewness of intensity, histogram and contrast, correlation, dissimilarity, energy, entropy, homogeneity, variance of gray level cooccurrence matrix 25 . These 16 features may help imaging physicians to make diagnostic decisions.

The statistical results were with the data obtained from reviewer $1(\mathrm{YW})$. To assess the consistency between reviewers, the data from 20 patients were randomized and analyzed again by another imaging physician without knowing the results (reviewer 2: JD). Both reviewers with more than 10 years of experience in abdominal MR imaging were blinded to the final diagnosis. There were no missing data and no adverse event.

\section{Statistical analysis}

The Kolmogorov-Smirnov test tested the normal 

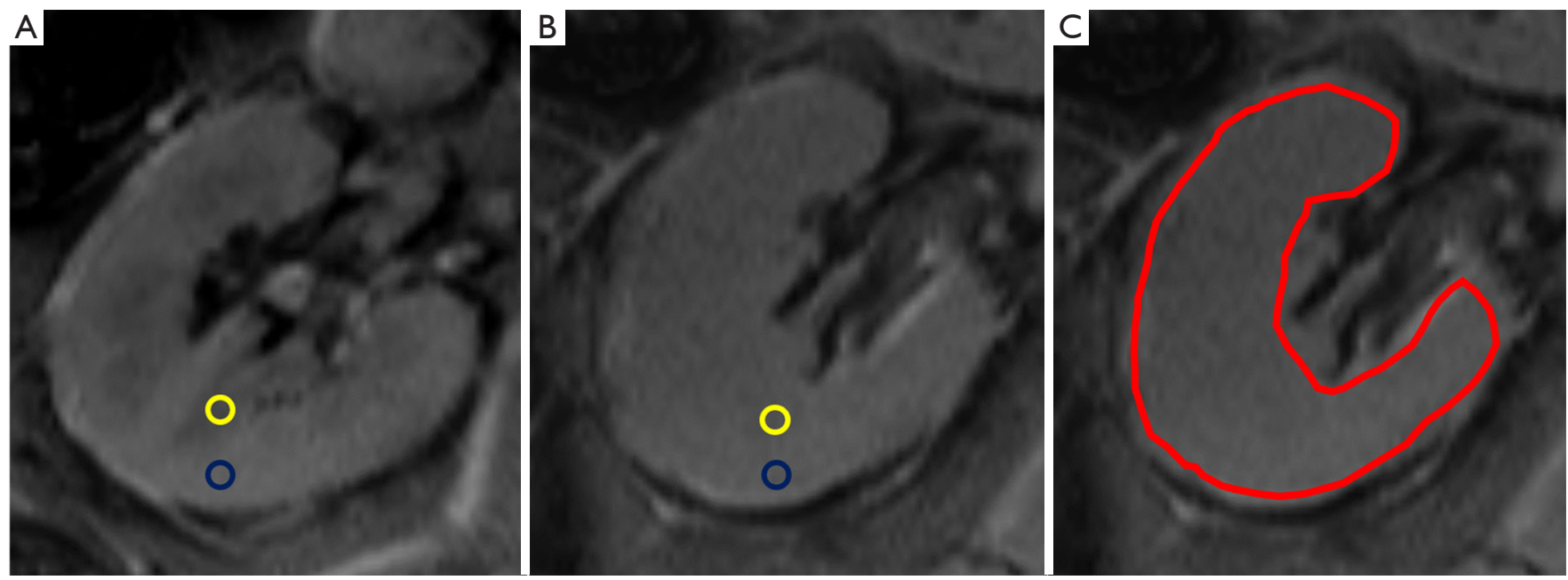

Figure 2 (A) A patient in non-msRI, male, 60 years old. The boundary between the cortex and medulla was clear on the fSWI image. ROIs were placed in the cortical region (blue) and medulla region (yellow), respectively. (B,C) A patient in a moderate-severe renal injured group, male, 50 years old. The boundary between the cortex and medulla was unclear on the fSWI image. The cortical ROI (blue) was placed close to the edge of the cortical region, and the medullary ROI (yellow) was placed in the middle of the renal parenchyma. The ROI used for texture analysis was drawn along the kidney outline (red). Non-msRI, non-moderate-severe renal injured group; fSWI, fusion susceptibilityweighted imaging; ROI, region-of-interest.

distribution of measurement data. The parametric data were expressed as $\bar{x} \pm \mathrm{s}$, and the median and interquartile range expressed non-parametric data. This frequency expresses the count data.

The consistency between different reviewers' assessments was tested by intraclass correlation efficient (ICC): ICC $<0.40$ was poor, ICC between 0.40 to 0.75 was good, and ICC $>0.75$ was excellent. A paired t-test within the nonmsRI or msRI groups is used to compare CPswi and MPswi. SWI parameters and texture features of non-msRI and msRI groups were compared using an independent sample $t$-test or Mann-Whitney $\mathrm{U}$ test. The receiver operating characteristic curve (ROC) was used to analyze and compare the diagnostic efficacy of the parameters for the msRI group. The ability of SWI parameters and texture features in predicting msRI was analyzed by binary logistic regression. The correlations between SWI parameters, texture features, and BUN, Scr were analyzed by Spearman: $|\mathrm{r}|<0.2$ was weakly correlated or uncorrelated, 0.2 to 0.4 was weakly correlated, 0.4 to 0.6 was moderately correlated, 0.6 to 0.8 was generally correlated, and $|r|>0.8$ was strongly correlated.

ROC curve analysis was conducted using Medcalc 18.9.1 (Ostend, Belgium) software, and other statistical analyses were performed with SPSS 25.0 (IBM, USA) software.
All statistics were two-sided, and $\mathrm{P}<0.05$ was considered statistically significant.

\section{Results}

\section{Clinical and laboratory characteristics}

Forty-five patients with T2DM (37 males, 8 females; mean age, $57 \pm 11$ years; range, $27-77$ years) were ultimately included. The patients were divided into two groups: non-msRI group $(n=22)$ and msRI group $(n=23)$. The typical characteristics of patients, including age, gender, hypertension, body mass index (BMI), BUN, Scr, and eGFR, are shown in Table 2.

\section{Inter-reviewer agreement}

Except for CMswi, whose inter-reviewer consistency was lower (ICC $=0.62$ ), all other SWI parameters and texture features showed substantial inter-reviewer consistency (ICC $>0.75$ ) (Table S1).

\section{Comparison of SWI parameters and texture features in intra- and inter-group}

Within each group (non-msRI and msRI group), MPswi 
Table 2 Patient clinical and laboratory data

\begin{tabular}{lcc}
\hline Clinical indexes & Non-msRI $(\mathrm{n}=22)$ & MsRI $(\mathrm{n}=23)$ \\
\hline Clinical data & & $58.26 \pm 8.83$ \\
Age (year) & $55.41 \pm 13.59$ & $20 / 23(86.9 \%)$ \\
Gender (male) & $17 / 22(77.2 \%)$ & $20 / 23(86.9 \%)$ \\
Hypertension $(+)$ & $17 / 22(77.2 \%)$ & $25.24(22.89,27.75)$ \\
BMI $\left(\mathrm{kg} / \mathrm{m}^{2}\right)$ & $25.01(24.01,27.42)$ & $14.20 \pm 6.82$ \\
Laboratory data & $5.40 \pm 1.56$ & $301.86 \pm 164.24$ \\
BUN $(\mathrm{mmol} / \mathrm{L})$ & $74.01 \pm 15.26$ & $21.83(12.00,36.28)$ \\
Scr $(\mu \mathrm{mol} / \mathrm{L})$ & $86.19(74.40,120.00)$ & \\
eGFR $\left(\mathrm{mL} / \mathrm{min} / 1.73 \mathrm{~m}^{2}\right)$ & \\
\hline
\end{tabular}

Non-msRI, non-moderate-severe renal injured; msRI, moderate-severe renal injured; BMI, body mass index; BUN, blood urea nitrogen; Scr, serum creatinine; eGFR, estimated glomerular filtration rate.

was significantly lower than CPswi $(t=8.619,3.483$, respectively, $\mathrm{P}<0.05)$. When comparing the two groups, the msRI group showed higher MPswi, and lower CMswi, Skewness, Correlation $(\mathrm{P}<0.05)$ (Table 3).

\section{Differentiation between non-msRI and msRI group}

MPswi, CMswi, Skewness and Correlation could distinguish between msRI group and non-msRI group (AUC $=0.703$, $0.854,0.754,0.708$, respectively) (Table 4). The diagnostic abilities were similar among these parameters $(\mathrm{Z}=0.055$ $1.669, \mathrm{P}>0.05$ ) (Figure 3).

\section{The protection factors of the msRI group}

MPswi, CMswi, Skewness, and Correlation were transformed into binary categorical variables according to the cutoff values shown in Table 4 and then analyzed by logistic regression. The results showed that CMswi $(\leq 1.117=0,>1.117=1)$ was an independent protective factor of msRI $(\mathrm{OR}=0.026,95 \% \mathrm{CI}=0.002-0.279, \mathrm{P}=0.003)$ (Table S2).

\section{Correlation between SWI parameters, textual features and BUN, Scr}

MPswi and CMswi are moderately correlated with BUN $(\mathrm{r}=0.416, \mathrm{P}=0.004 ; \mathrm{r}=-0.545, \mathrm{P}<0.001)$. CMswi was strongly correlated with $\mathrm{Scr}(\mathrm{r}=-0.645, \mathrm{P}<0.001)$. Correlation was moderately correlated with $\mathrm{Scr}(\mathrm{r}=-0.411, \mathrm{P}=0.005)$. MPswi,
MeanAD, Skewness and Variance were weakly correlated with $\operatorname{Scr}(\mathrm{r}=0.363,-0.305,-0.395,-0.337, \mathrm{P}=0.014,0.042$, $0.007,0.023$, respectively).

\section{Discussion}

Chronic hypoxic injury is considered a common pathway for end-stage renal disease (3). SWI can reflect the tissue blood oxygen level and supply an oxygen metabolism index for understanding the mechanism of renal injury and monitoring drug efficacy. Thus, in this study, we examined the SWI parameters and SWI-based texture features of kidneys in patients with T2DM, to verify its performance in evaluating renal dysfunction.

SWI can evaluate renal function. Lower blood oxygen levels usually indicate more deoxyhemoglobin and a more significant difference in susceptibility between blood vessels and the surrounding tissues (16). Therefore, SWI can reflect tissue blood oxygen levels. In studies of iron abnormality in cirrhotic livers, the SWI phase was negatively correlated with R2* (17). Another study showed that, as the liver fat accumulated, the SWI signal and $\mathrm{T} 2{ }^{*}$ relaxation time significantly decreased (18). Mie et al. (19) first applied SWI technology to kidney study. Ding et al. (6) showed that both the renal medulla phase value and $\mathrm{T}^{*}$ increase after water load; thus, SWI can monitor medullary blood oxygen levels after water load. Since SWI does not produce direct value, we used a signal intensity ratio technique to analyze the data. In our study, MPswi, CMswi, Skewness, and Correlation could monitor renal injury. 
Table 3 Comparison between non-msRI group and msRI group of SWI parameters and texture features

\begin{tabular}{|c|c|c|c|c|}
\hline Variable & Non-msRI (n=22) & MsRI (n=23) & $t / u$ & $P$ value \\
\hline CPswi & $1.16 \pm 0.24$ & $1.22 \pm 0.26$ & -0.85 & 0.39 \\
\hline MPswi & $0.96 \pm 0.21$ & $1.16 \pm 0.27$ & -2.65 & $0.01^{*}$ \\
\hline CMswi & $1.19(1.10,1.29)$ & $1.06(0.99,1.09)$ & 74.00 & $<0.001^{*}$ \\
\hline IQR & $49.15 \pm 11.39$ & $45.87 \pm 13.89$ & 0.86 & 0.39 \\
\hline Kurtosis & $2.89(2.58,4.21)$ & $2.95(2.53,3.35)$ & 256.0 & 0.94 \\
\hline MeanAD & $27.67 \pm 6.14$ & $26.00 \pm 7.40$ & 0.82 & 0.41 \\
\hline MedianAD & $23.79 \pm 5.58$ & $22.76 \pm 6.82$ & 0.55 & 0.58 \\
\hline P75 & $197.50(174.00,228.50)$ & $211.00(174.00,230.00)$ & 251.00 & 0.96 \\
\hline Range & $232.23 \pm 71.89$ & $216.39 \pm 62.18$ & 0.79 & 0.43 \\
\hline Skewness & $0.23(-0.09,0.63)$ & $-0.08(-0.46,0.12)$ & 124.50 & $<0.001^{*}$ \\
\hline \multicolumn{5}{|l|}{ GLCM features } \\
\hline Contrast & $8.01(6.23,13.29)$ & $12.43(6.89,20.03)$ & 321.00 & 0.12 \\
\hline Correlation & $0.89(0.85,0.92)$ & $0.83(0.72,0.90)$ & 147.50 & $0.01^{*}$ \\
\hline Dissimilarity & $1.94(1.79,2.61)$ & $2.73(1.70,3.53)$ & 329.00 & 0.08 \\
\hline Energy & $0.006(0.004,0.008)$ & $0.006(0.004,0.007)$ & 223.50 & 0.49 \\
\hline
\end{tabular}

*, $\mathrm{P}<0.05$. Non-msRI, non-moderate-severe renal injured group; msRI, moderate-severe renal injured group; SWI, susceptibilityweighted imaging; GLCM, GrayLevelCooccurenceMatrix; CPswi, the signal intensity ratio of cortex to psoas muscle; MPswi, the signal intensity ratio of medulla to psoas muscle; CMswi, the signal intensity ratio of cortex to medulla; IQR, InterQuartileRange; MeanAD, MeanAbsoluteDeviation; MedianAD, MedianAbsoluteDeviation.

Table 4 ROC analysis of using SWI parameters and texture features to identify the msRI group

\begin{tabular}{lcccccccc}
\hline Indexes & AUC & P & Cutoff value & Sensibility (\%) & Specificity (\%) & Accuracy (\%) & +LR & -LR \\
\hline MPswi & 0.703 & 0.0109 & 1.006 & 73.91 & 63.64 & 70.9 & 2.03 & 0.41 \\
CMswi & 0.854 & $<0.0001$ & 1.117 & 95.65 & 82.61 & 59.09 & 82.3 & 3.51 \\
Skewness & 0.754 & 0.0005 & 0.144 & 0.06 & 68.9 & 2.02 & 0.29 \\
Correlation & 0.708 & 0.0086 & 0.855 & 65.22 & 77.27 & 68.9 & 2.87 & 0.45 \\
\hline
\end{tabular}

SWI, susceptibility-weighted imaging; msRI, the moderate-severe renal injured group; MPswi, the signal intensity ratio of the medulla to psoas muscle; CMswi, the signal intensity ratio of the cortex to medulla; AUC, the area under the curve; LR, the likelihood ratio. 


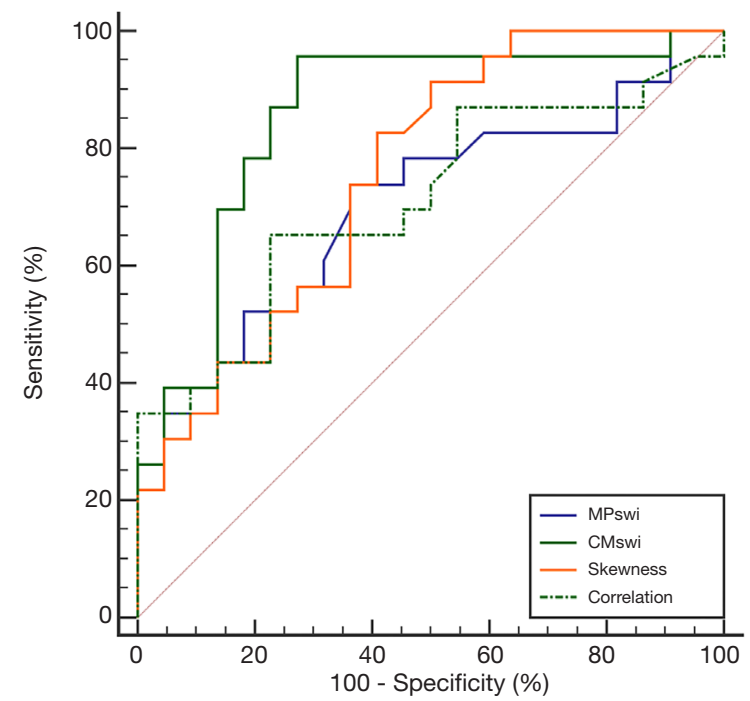

Figure 3 Receiver operating characteristic curves of selected SWI parameters and texture features in distinguishing between non-msRI and msRI. The diagnostic abilities of MPswi, CMswi, Skewness and Correlation were similar $(\mathrm{Z}=0.055-1.669, \mathrm{P}>0.05)$. Non-msRI, non-moderate-severe renal injured group; msRI, moderate-severe renal injured group; SWI, susceptibility-weighted imaging; MPswi, the signal intensity ratio of the medulla to psoas muscle; CMswi, the signal intensity ratio of the cortex to the medulla.

We found MPswi was significantly lower than CPswi in both non-msRI and msRI groups. We hypothesized that this signal change came from the increased deoxyhemoglobin level in the medulla so that its blood oxygen level was lower than the cortex. This result is consistent with most previous studies on blood oxygen level-dependent MRI (BOLDMRI) in humans and animals (20-23). Because of the oxygen consumption from renal tubular mediated $\mathrm{Na}$ ion reabsorption (3), and the oxygen shunting from medullary arterial-venous counter-flow (24), the kidney medulla shows physiological hypoxia, which is necessary for the development of kidney injury $(25,26)$. In diabetic patients, the glomerular hyperfiltration increases oxygen delivery, and the sodium-glucose cotransporter mediates the increase of sodium and glucose reabsorption in the renal tubular, which further enhances oxygen consumption $(2,27)$. This positive feedback circle leads to increased oxygen demand. However, hyperglycemia attenuates the hypoxia-inducible factor- $1 \alpha$ (HIF-1 $\alpha$ ) mediated response to hypoxia, and indirectly changes the hemodynamic or/and metabolism in diabetic kidneys (2). Both hyperfiltration and hyperglycemia can lead to hypoxia in diabetic kidneys and decrease blood oxygen levels.

In the moderate-severe renal injured cases, there was no notable change in cortical signal intensity when compared to the non-msRI group, but the medullary signal intensity was significantly increased. These results might be because of the decreased deoxygenated hemoglobin in the medulla of the msRI group, which caused the increase of blood oxygen levels. Previous studies have found that, in the rabbit unilateral ureteral obstruction model, as the kidney damage increased and the fibrotic area expanded, the cortical and medullary SWI signal decreased (7), and the blood oxygen level gradually decreased (28). However, in patients with diabetic nephropathy, the changes in renal blood oxygen levels are more complicated. Similar R2* studies could not get consistent results in CKD patients. Wang et al. (21) and Milani et al. (29) reported that the medullary blood oxygen levels increased in CKD patients, which might be because of the decreased eGFR and reabsorption of medullary material; the reduced oxygen consumption then caused increased blood oxygen levels. However, other studies showed that the medullary blood oxygen levels in CKD patients were lower than normal people $(22,30)$, suggesting that the medullary blood flow is insufficient, and is more susceptible to ischemia. There are also studies showing that the medullary blood oxygen levels in CKD patients were like the control group (23,31-33), and the cortical blood oxygen levels in CKD patients were unchanged or even increased (21,30-34). The inconsistency between the results may be because of different local deoxygenated hemoglobin concentrations, and the variations in hydration status, blood $\mathrm{pH}$, hematocrit, and magnetic sensitivity (35).

MRI texture analysis can ease the analysis and evaluation of renal function. All images comprise pixels, which are grayscale units within the defined range, representing the points in space (9). We performed the first and secondorder statistical analyses on fSWI images. The firstorder analysis used a histogram to analyze the intensity of each pixel, and the second-order analysis used a gray level co-occurrence matrix (GLCM) to analyze the interrelationship or co-occurrence between pixel intensities. We found the Skewness and Correlation were significantly lower in the msRI group compared to the non-msRI group. Skewness is a measurement for histogram asymmetry, and zero represents the normal distribution (36). Correlation is a measurement for the probability of specified pixel pairs occurring together (36). We speculated that the decreased deoxyhemoglobin content might cause these changes 
with increased SWI signal intensity in the medulla of the msRI group so that the heterogeneity between cortical and medullary pixels was lower than the non-msRI group. A recent study (11) reported the texture features with DWI, BOLD, and SWI could test renal dysfunction, and EntropyBOLD and CorrelationSWI can help assess early renal dysfunction. Also, a discriminant equation with GLCM parameters of BOLD-MRI can predict the renal pathology of lupus nephritis (37). Texture features are an important prognostic biomarker for the progression of renal degeneration in autosomal dominant polycystic kidney disease (10). In these studies, texture analysis shows higher diagnostic and predictive value.

This study also has some limitations. First, the sample size is small, and there might be a sampling bias. Second, only the right kidney was measured. Although most previous studies found no significant differences in blood oxygen levels in both kidneys (38-40), and the gastrointestinal air affects left kidney image, single kidney analysis may still cause measurement bias. Third, we adopted the most widely used ROI measurement method. Some alternative methods have been used for renal function analysis, including compartmental approach, fractional hypoxia method, concentric object method, and twelvelayer concentric object method, but there is no consensus on which method is better (41). Fourth, many challenges still exist for applying texture analysis to clinical and research applications. Some unrelated lesion factors can independently affect the results of texture analysis, for example, the slice thickness, voxels, gray levels, noise, and resolution of the images (9).

In conclusion, from the SWI imaging, we found MPswi is higher, and CMswi, Skewness, Correlation are lower in the moderate-severe renal injured group. Although this study found that SWI parameters could reflect changes in medullary oxygenation levels, the mechanism of increased medullary SWI signals corresponding to high oxygenation levels is yet to be explored by animal experiments. Texture analysis provided us with more information about the heterogeneity of cortical and medulla signals that could not be observed with naked eyes, opening a new window for studying renal impairment.

\section{Acknowledgments}

Funding: The study was supported by the National Natural Science Foundation of China (81771798, 81901696) and Applied Basic Research Program of Changzhou

\section{(CJ20180069).}

\section{Footnote}

Reporting Checklist: The authors have completed the STARD reporting checklist. Available at http://dx.doi.org/10.21037/ atm-20-7121

Data Sharing Statement: Available at http://dx.doi. org/10.21037/atm-20-7121

Conflicts of Interest: All authors have completed the ICMJE uniform disclosure form (available at http://dx.doi. org/10.21037/atm-20-7121). The authors have no conflicts of interest to declare.

Ethical Statement: The authors are accountable for all aspects of the work in ensuring that questions related to the accuracy or integrity of any part of the work are appropriately investigated and resolved. All procedures performed in this study involving human participants were in accordance with the Declaration of Helsinki (as revised in 2013). The study was approved by the ethics board of the third affiliated hospital of Soochow University (No. 2016$010)$ and informed consent was taken from all the patients.

Open Access Statement: This is an Open Access article distributed in accordance with the Creative Commons Attribution-NonCommercial-NoDerivs 4.0 International License (CC BY-NC-ND 4.0), which permits the noncommercial replication and distribution of the article with the strict proviso that no changes or edits are made and the original work is properly cited (including links to both the formal publication through the relevant DOI and the license). See: https://creativecommons.org/licenses/by-nc-nd/4.0/.

\section{References}

1. Thomas MC, Cooper ME, Zimmet P. Changing epidemiology of type 2 diabetes mellitus and associated chronic kidney disease. Nat Rev Nephrol 2016;12:73-81.

2. Takiyama Y, Haneda M. Hypoxia in diabetic kidneys. Biomed Res Int 2014;2014:837421.

3. Hansell P, Welch WJ, Blantz RC, et al. Determinants of kidney oxygen consumption and their relationship to tissue oxygen tension in diabetes and hypertension. Clin Exp Pharmacol Physiol 2013;40:123-37.

4. Fu H, Liu S, Bastacky SI, et al. Diabetic kidney diseases 
revisited: A new perspective for a new era. Mol Metab 2019;30:250-63.

5. Liu S, Buch S, Chen Y, et al. Susceptibility-weighted imaging: current status and future directions. Nmr Biomed 2017;30:e3552.

6. Ding J, Xing W, Wu D, et al. Evaluation of Renal Oxygenation Level Changes after Water Loading Using Susceptibility-Weighted Imaging and T2* Mapping. Korean J Radiol 2015;16:827-34.

7. Zhang JG, Xing ZY, Zha TT, et al. Longitudinal assessment of rabbit renal fibrosis induced by unilateral ureteral obstruction using two-dimensional susceptibility weighted imaging. J Magn Reson Imaging 2018;47:1572-7.

8. Sun J, Yu S, Chen J, et al. Assessment of delayed graft function using susceptibility-weighted imaging in the early period after kidney transplantation: a feasibility study. Abdom Radiol (NY) 2019;44:218-26.

9. Thomas R, Qin L, Alessandrino F, et al. A review of the principles of texture analysis and its role in imaging of genitourinary neoplasms. Abdom Radiol (NY) 2019;44:2501-10.

10. Kline TL, Korfiatis P, Edwards ME, et al. Image texture features predict renal function decline in patients with autosomal dominant polycystic kidney disease. Kidney Int 2017;92:1206-16.

11. Ding J, Xing Z, Jiang Z, et al. Evaluation of renal dysfunction using texture analysis based on DWI, BOLD, and susceptibility-weighted imaging. Eur Radiol 2019;29:2293-301.

12. American DA. Diagnosis and classification of diabetes mellitus. Diabetes Care 2010;33 Suppl 1:S62-9.

13. Schwandt A, Denkinger M, Fasching P, et al. Comparison of MDRD, CKD-EPI, and Cockcroft-Gault equation in relation to measured glomerular filtration rate among a large cohort with diabetes. J Diabetes Complications 2017;31:1376-83.

14. KDOQI Clinical Practice Guidelines and Clinical Practice Recommendations for Diabetes and Chronic Kidney Disease. Am J Kidney Dis 2007;49:S12-154.

15. Zhang L, Fried DV, Fave XJ, et al. IBEX: an open infrastructure software platform to facilitate collaborative work in radiomics. Med Phys 2015;42:1341-53.

16. Bauer WR, Nadler W, Bock $M$, et al. Theory of the BOLD effect in the capillary region: an analytical approach for the determination of T2 in the capillary network of myocardium. Magn Reson Med 1999;41:51-62.

17. Tao R, Zhang J, Dai Y, et al. An in vitro and in vivo analysis of the correlation between susceptibility-weighted imaging phase values and R2* in cirrhotic livers. Plos One 2012;7:e45477.

18. Obmann VC, Marx C, Berzigotti A, et al. Liver MRI susceptibility-weighted imaging (SWI) compared to T2* mapping in the presence of steatosis and fibrosis. Eur J Radiol 2019;118:66-74.

19. Mie MB, Nissen JC, Zollner FG, et al. Susceptibility weighted imaging (SWI) of the kidney at 3T-initial results. Z Med Phys 2010;20:143-50.

20. Prasad P, Li LP, Halter S, et al. Evaluation of renal hypoxia in diabetic mice by BOLD MRI. Invest Radiol 2010;45:819-22.

21. Wang ZJ, Kumar R, Banerjee S, et al. Blood oxygen level-dependent (BOLD) MRI of diabetic nephropathy: preliminary experience. J Magn Reson Imaging 2011;33:655-60.

22. Yin WJ, Liu F, Li XM, et al. Noninvasive evaluation of renal oxygenation in diabetic nephropathy by BOLDMRI. Eur J Radiol 2012;81:1426-31.

23. Prasad PV, Thacker J, Li LP, et al. Multi-Parametric Evaluation of Chronic Kidney Disease by MRI: A Preliminary Cross-Sectional Study. PLoS One 2015;10:e0139661.

24. Zhang JL, Morrell G, Rusinek H, et al. Measurement of renal tissue oxygenation with blood oxygen leveldependent MRI and oxygen transit modeling. Am J Physiol Renal Physiol 2014;306:F579-87.

25. Hirakawa Y, Tanaka T, Nangaku M. Renal Hypoxia in CKD; Pathophysiology and Detecting Methods. Front Physiol 2017;8:99.

26. Mimura I, Nangaku M. The suffocating kidney: tubulointerstitial hypoxia in end-stage renal disease. Nat Rev Nephrol 2010;6:667-78.

27. Jerums G, Premaratne E, Panagiotopoulos S, et al. The clinical significance of hyperfiltration in diabetes. Diabetologia 2010;53:2093-104.

28. Zha T, Ren X, Xing Z, et al. Evaluating Renal Fibrosis with R2* Histogram Analysis of the Whole Cortex in a Unilateral Ureteral Obstruction Model. Acad Radiol 2019;26:e202-7.

29. Milani B, Ansaloni A, Sousa-Guimaraes S, et al. Reduction of cortical oxygenation in chronic kidney disease: evidence obtained with a new analysis method of blood oxygenation level-dependent magnetic resonance imaging. Nephrol Dial Transplant 2017;32:2097-105.

30. Xin-Long P, Jing-Xia X, Jian-Yu L, et al. A preliminary study of blood-oxygen-level-dependent MRI in patients 
with chronic kidney disease. Magn Reson Imaging 2012;30:330-5.

31. Michaely HJ, Metzger L, Haneder S, et al. Renal BOLDMRI does not reflect renal function in chronic kidney disease. Kidney Int 2012;81:684-9.

32. Pruijm M, Hofmann L, Piskunowicz M, et al. Determinants of renal tissue oxygenation as measured with BOLD-MRI in chronic kidney disease and hypertension in humans. Plos One 2014;9:e95895.

33. Thacker JM, Li LP, Li W, et al. Renal Blood Oxygenation Level-Dependent Magnetic Resonance Imaging: A Sensitive and Objective Analysis. Invest Radiol 2015;50:821-7.

34. Inoue $\mathrm{T}$, Kozawa $\mathrm{E}, \mathrm{Okada} \mathrm{H}$, et al. Noninvasive evaluation of kidney hypoxia and fibrosis using magnetic resonance imaging. J Am Soc Nephrol 2011;22:1429-34.

35. Neugarten J, Golestaneh L. Blood oxygenation leveldependent MRI for assessment of renal oxygenation. Int J Nephrol Renovasc Dis 2014;7:421-35.

36. Khene ZE, Bensalah K, Largent A, et al. Role of quantitative computed tomography texture analysis in the prediction of adherent perinephric fat. World J Urol 2018;36:1635-42.

Cite this article as: Jiang Z, Wang Y, Ding J, Yu S, Zhang J, Zhou H, Di J, Xing W. Susceptibility weighted imaging (SWI) for evaluating renal dysfunction in type 2 diabetes mellitus: a preliminary study using SWI parameters and SWI-based texture features. Ann Transl Med 2020;8(24):1673. doi: 10.21037/ atm-20-7121
37. Shi H, Jia J, Li D, et al. Blood oxygen level-dependent magnetic resonance imaging for detecting pathological patterns in patients with lupus nephritis: a preliminary study using gray-level co-occurrence matrix analysis. J Int Med Res 2018;46:204-18.

38. Hofmann L, Simon-Zoula S, Nowak A, et al. BOLDMRI for the assessment of renal oxygenation in humans: acute effect of nephrotoxic xenobiotics. Kidney Int 2006;70:144-50.

39. Leung G, Kirpalani A, Szeto SG, et al. Could MRI Be Used To Image Kidney Fibrosis? A Review of Recent Advances and Remaining Barriers. Clin J Am Soc Nephrol 2017;12:1019-28.

40. Li C, Liu H, Li X, et al. Application of BOLD-MRI in the classification of renal function in chronic kidney disease. Abdom Radiol (NY) 2019;44:604-11.

41. Chen F, Li S, Sun D. Methods of Blood Oxygen LevelDependent Magnetic Resonance Imaging Analysis for Evaluating Renal Oxygenation. Kidney Blood Press Res 2018;43:378-88.

(English Language Editor: J. Chapnick) 
Supplementary

Table S1 The inter-reviewer consistency of SWI parameters and texture features

\begin{tabular}{|c|c|c|}
\hline \multirow{2}{*}{ Variable } & \multicolumn{2}{|c|}{ ICC } \\
\hline & $r$ & $\mathrm{P}$ \\
\hline \multicolumn{3}{|l|}{ SWI parameters } \\
\hline CPswi & 0.87 & $<0.001$ \\
\hline MPswi & 0.87 & $<0.001$ \\
\hline CMswi & 0.62 & 0.001 \\
\hline \multicolumn{3}{|c|}{ Histogram features } \\
\hline IQR & 0.96 & $<0.001$ \\
\hline Kurtosis & 0.75 & $<0.001$ \\
\hline MeanAD & 0.97 & $<0.001$ \\
\hline MedianAD & 0.97 & $<0.001$ \\
\hline P25 & 0.99 & $<0.001$ \\
\hline P50 & 0.99 & $<0.001$ \\
\hline P75 & 0.99 & $<0.001$ \\
\hline Range & 0.90 & $<0.001$ \\
\hline Skewness & 0.84 & $<0.001$ \\
\hline \multicolumn{3}{|l|}{ GLCM features } \\
\hline Contrast & 0.97 & $<0.001$ \\
\hline Correlation & 0.98 & $<0.001$ \\
\hline Dissimilarity & 0.99 & $<0.001$ \\
\hline Energy & 0.98 & $<0.001$ \\
\hline Entropy & 0.98 & $<0.001$ \\
\hline Homogeneity & 0.99 & $<0.001$ \\
\hline Variance & 0.97 & $<0.001$ \\
\hline
\end{tabular}

ICC, intraclass correlation coefficient; SWI, susceptibilityweighted imaging; GLCM, GrayLevelCooccurenceMatrix; IQR, InterQuartileRange; MeanAD, MeanAbsoluteDeviation; MedianAD, MedianAbsoluteDeviation.
Table S2 Multivariate regression analysis on MPswi, CMswi, skewness, and correlation for the prediction of moderate to severe renal injury

\begin{tabular}{lcc}
\hline Indexes & OR $(95 \% \mathrm{Cl})$ & P value \\
\hline MPswi & $2.429(0.388,15.213)$ & 0.343 \\
CMswi & $0.026(0.002,0.279)$ & 0.003 \\
Skewness & $4.192(0.667,26.345)$ & 0.126 \\
Correlation & $0.383(0.065,2.239)$ & 0.287 \\
\hline
\end{tabular}

OR, the odds ratio; MPswi, the signal intensity ratio of the medulla to psoas muscle; CMswi, the signal intensity ratio of the cortex to the medulla. 